This item was submitted to Loughborough's Research Repository by the author.

Items in Figshare are protected by copyright, with all rights reserved, unless otherwise indicated.

\title{
Secure full-duplex two-way relaying for SWIPT
}

PLEASE CITE THE PUBLISHED VERSION

https://doi.org/10.1109/LWC.2017.2776928

PUBLISHER

IEEE

VERSION

VoR (Version of Record)

\section{PUBLISHER STATEMENT}

This work is made available according to the conditions of the Creative Commons Attribution 3.0 Unported (CC BY 3.0) licence. Full details of this licence are available at: http://creativecommons.org/licenses/by/3.0/

\section{LICENCE}

CC BY 3.0

\section{REPOSITORY RECORD}

Okandeji, Alexander A., Muhammad R. Khandaker, Kai-Kit Wong, Gan Zheng, Yangyang Zhang, and Zhongbin Zheng. 2019. "Secure Full-duplex Two-way Relaying for SWIPT". figshare.

https://hdl.handle.net/2134/28149. 


\title{
Secure Full-Duplex Two-Way Relaying for SWIPT
}

\author{
Alexander A. Okandeji ${ }^{\circledR}$, Muhammad R. A. Khandaker ${ }^{\circledR}$, Kai-Kit Wong, Gan Zheng, \\ Yangyang Zhang, and Zhongbin Zheng
}

\begin{abstract}
This letter studies bi-directional secure information exchange in a simultaneous wireless information and power transfer system enabled by a full-duplex multiple-input multipleoutput amplify-and-forward (AF) relay. The AF relay injects artificial noise (AN) in order to confuse the eavesdropper. Specifically, we assume a zeroforcing (ZF) solution constraint to eliminate the residual self-interference. As a consequence, we address the optimal joint design of the $\mathrm{ZF}$ matrix and the $\mathrm{AN}$ covariance matrix at the relay node as well as the transmit power at the sources. We propose an alternating algorithm utilizing semi-definite programming technique and 1-D searching to achieve the optimal solution. Simulation results are provided to demonstrate the effectiveness of the proposed algorithm.
\end{abstract}

Index Terms-Full-duplex, energy harvesting, relay, secrecy, physical layer security.

\section{INTRODUCTION}

W IRELESS communication networks are inherently susceptible to sophisticated eavesdropping attacks; however, full-duplex wireless operation is even more prone to overhearing due to continuous transmission and reception by the full-duplex nodes. Guaranteeing security in full-duplex communications is therefore a critical issue that needs to be addressed.

Recently, simultaneous wireless information and power transfer (SWIPT) [1], full-duplex (FD) enabled bi-directional wireless communications [2] as well as physical-layer (PHY) security [3] have each been a major research area and also led to efforts investigating the combination of these technologies. To name a few, for example, FD SWIPT has been considered in [4]. Also, PHY security in FD systems was addressed in [5] and [6]. In contrast to existing works, our main contribution is the study of the integration of all three and the joint optimization of the transmit power, the artificial noise (AN) covariance and the two-way relay beamforming matrix to maximize the secrecy sum-rate for SWIPT with a FD

Manuscript received August 3, 2017; revised October 15, 2017; accepted November 8, 2017. Date of publication November 23, 2017; date of current version June 19, 2018. This work was supported in part by the Presidential Special Scholarship Scheme for Innovation and Development, Federal Republic of Nigeria, and in part by the EPSRC under Grant EP/N008219/1. The work of G. Zheng was supported by the U.K. EPSRC under Grant EP/N007840/1. The associate editor coordinating the review of this paper and approving it for publication was I. Krikidis. (Corresponding author: Muhammad R. A. Khandaker.)

A. A. Okandeji, M. R. A. Khandaker, and K.-K. Wong are with the Department of Electronic and Electrical Engineering, University College London, London WC1E 7JE, U.K. (e-mail: alexander.okandeji.13@ucl.ac.uk; m.khandaker@ucl.ac.uk).

G. Zheng is with the Wolfson School of Mechanical, Electrical and Manufacturing Engineering, Loughborough University, Loughborough, LE11 3TU, U.K.

Y. Zhang is with the Kuang-Chi Institute of Advanced Technology, Shenzhen 518057, China.

Z. Zheng is with the East China Institute of Telecommunications, China Academy of Information and Communications Technology, Shanghai 100191, China.

Digital Object Identifier 10.1109/LWC.2017.2776928 multiple-input multiple-output (MIMO) amplify-and-forward (AF) relay employing power splitter (PS). Specifically, the total transmit power is minimized while guaranteeing the signal to interference and noise ratio (SINR) constraints at the two legitimate users as well as the eavesdropper and the energy harvesting constraint at the relay.

Notations: We use $\mathbf{X} \in \mathbb{C}^{M \times N}$ to represent a complex $M \times N$ matrix. Also, $(\cdot)^{\dagger}$ denotes the conjugate transpose, trace $(\cdot)$ is the trace operation, and $\|\cdot\|$ denotes the Frobenius norm. In addition, $|\cdot|$ returns the absolute value of a scalar, and $\mathbf{X} \succeq \mathbf{0}$ denotes that the Hermitian matrix $\mathbf{X}$ is positive semidefinite. The expectation operator is denoted by $\mathbb{E}\{\cdot\}$.

\section{SySTEM MODEL}

We consider SWIPT in a three-node MIMO relay network with nodes $\mathrm{S}_{\mathrm{A}}$ and $\mathrm{S}_{\mathrm{B}}$, equipped with one transmit and one receive antennas for information transmission and reception, respectively, exchanging confidential information with the aid of a multi-antenna AF relay $R$, in the presence of a single antenna eavesdropper $E$. We assume that: i) $S_{A}, S_{B}$ and $R$ all operate in FD mode, ii) there is no direct link between $S_{A}$ and $\mathrm{S}_{\mathrm{B}}$, iii) the harvested energy at the relay is used to complete the bi-directional information exchange [7], and iv) $S_{A}$ and $S_{B}$ are not aware of any eavesdropper thus, no direct link exist between $S_{A}$ and $S_{B}$ and the eavesdropper, as in [8]. The inclusion of the source-eavesdropper links can be handled in a similar manner. The relay, however, is aware of the eavesdropper. As a result, the relay injects AN signals to confuse the eavesdropper.

In the first phase, the relay receives confidential information from $S_{A}$ and $S_{B}$, while in the next phase, $R$ amplifies and forwards the processed information to both nodes with the AN signal being superimposed to jam the eavesdropper [8]. Using the transmit power $P_{A}$ and $P_{B}$, respectively, $\mathrm{S}_{\mathrm{A}}$ and $\mathrm{S}_{\mathrm{B}}$ transmit their confidential messages simultaneously to $R$. On the other hand, $\mathrm{R}$ employs linear processing with amplification matrix $\mathbf{W}$ to process the received signal and broadcasts the processed signal to the nodes with harvested power U.

The antennas at $\mathrm{R}$ are separated for transmission and reception with $M_{T}$ transmit antennas and $M_{R}$ receive antennas. Also, we denote $\mathbf{h}_{X R} \in \mathbb{C}^{M_{R} \times 1}$ and $\mathbf{h}_{R X} \in \mathbb{C}^{M_{T} \times 1}$ to, respectively, represent the directional channel vectors between the node $\mathrm{X}$ $\in\{A, B\}$ and $\mathrm{R}$. Similarly, we use $\mathbf{h}_{R E}$ to denote the channel between $\mathrm{E}$ and $\mathrm{R}$.

To achieve FD communication, self-interference (SI) must be significantly suppressed, as total cancellation is not possible as a result of imperfect channel estimation [4]. Therefore, we adopt the use of existing SI cancellation mechanisms (e.g., antenna isolation, digital and analog cancellation, etc.), to reduce the effect of SI. For convenience, we denote $h_{A A}, h_{B B}$, and $\mathbf{H}_{R R} \in \mathbb{C}^{M_{R} \times M_{T}}$ as the residual SI (RSI) channels at the respective nodes [4]. Also, the RSI channel is represented as a Gaussian distribution random variable with zero mean and variance $\sigma_{X}^{2}$, for $X \in\{A, B, R\}$ [4]. Furthermore, the relay,

This work is licensed under a Creative Commons Attribution 3.0 License. For more information, see http://creativecommons.org/licenses/by/3.0/ 
assumed to be equipped with a PS device, coordinates information decoding and energy harvesting. Specifically, the relay splits the received signal power such that a $\rho \in(0,1)$ portion of the received signal power is fed to the information receiver (IR) and the remaining $(1-\rho)$ portion of the power is fed to the energy receiver (ER) at the relay.

\section{A. Signal Model}

The received signal $\mathbf{y}_{r}[n]$ and the transmit signal $\mathbf{x}_{R}[n]$ at $\mathrm{R}$ at time instant $n$, can be written, respectively, as

$$
\begin{aligned}
\mathbf{y}_{r}[n] & =\mathbf{h}_{A R} s_{A}[n]+\mathbf{h}_{B R} s_{B}[n]+\mathbf{H}_{R R} \mathbf{x}_{R}[n]+\mathbf{n}_{R}[n], \\
\mathbf{x}_{R}[n] & =\mathbf{W y}_{R}^{I R}[n-\tau]+\mathbf{z}[n],
\end{aligned}
$$

where $\tau$ is the processing delay to implement FD operation and assumed short enough to be neglected as far as the achievable rate computation is concerned, $\mathbf{n}_{R} \backsim \mathcal{C} \mathcal{N}\left(\mathbf{0}, \sigma_{R}^{2} \mathbf{I}\right)$ is the additive white Gaussian noise (AWGN) at $\mathbf{R}, \mathbf{z}[n] \backsim \mathcal{C N}(\mathbf{0}, \mathbf{Q})$, with $\mathbf{Q} \succeq \mathbf{0}$, is the AN used for interfering $\mathbf{E}$, and $\mathbf{y}_{R}^{I R}[n]$ is the signal split to the IR at $R$ given by

$$
\mathbf{y}_{R}^{I R}[n]=\sqrt{\rho}\left(\mathbf{h}_{A R} s_{A}[n]+\mathbf{h}_{B R} s_{B}[n]+\mathbf{H}_{R R} \mathbf{x}_{R}[n]+\mathbf{n}_{R}[n]\right) .
$$

Thus, the signal transmitted by $\mathrm{R}$ can then be expressed as

$$
\begin{aligned}
\mathbf{x}_{R}[n]= & \sqrt{\rho \mathbf{W}}\left(\mathbf{h}_{A R} s_{A}[n-\tau]+\mathbf{h}_{B R} s_{B}[n-\tau]\right. \\
& \left.+\mathbf{H}_{R R} \mathbf{x}_{R}[n-\tau]+\mathbf{n}_{R}[n-\tau]\right)+\mathbf{z}[n-\tau] .
\end{aligned}
$$

As shown in [9], the relay output can be further written as

$$
\begin{array}{r}
\mathbf{x}_{R}[n]=\mathbf{W} \sum_{j=0}^{\infty}\left(\mathbf{H}_{R R} \mathbf{W}\right)^{j}\left[\sqrt { \rho } \left(\mathbf{h}_{A R} s_{A}[n-j \tau-\tau]\right.\right. \\
\left.\left.+\mathbf{h}_{B R} s_{B}[n-j \tau-\tau]+\mathbf{n}_{R}[n-j \tau-\tau]\right)\right] \\
+\mathbf{z}[n-j \tau-\tau],
\end{array}
$$

where $j$ denotes the index of the delayed symbols. We define the covariance matrix of (5) as

$$
\begin{aligned}
\mathbb{E}\left[\mathbf{x}_{R} \mathbf{x}_{R}^{\dagger}\right]= & \rho\left[P_{A} \mathbf{W} \sum_{j=0}^{\infty}\left(\mathbf{H}_{R R} \mathbf{W}\right)^{j} \mathbf{h}_{A R} \mathbf{h}_{A R}^{\dagger}\left(\left(\mathbf{H}_{R R} \mathbf{W}\right)^{j}\right)^{\dagger} \mathbf{W}^{\dagger}\right. \\
& +P_{B} \mathbf{W} \sum_{j=0}^{\infty}\left(\mathbf{H}_{R R} \mathbf{W}\right)^{j} \mathbf{h}_{B R} \mathbf{h}_{B R}^{\dagger}\left(\left(\mathbf{H}_{R R} \mathbf{W}\right)^{j}\right)^{\dagger} \mathbf{W}^{\dagger} \\
& \left.+\mathbf{W} \sum_{j=0}^{\infty}\left(\mathbf{H}_{R R} \mathbf{W} \mathbf{W}^{\dagger} \mathbf{H}_{R R}^{\dagger}\right)^{j} \mathbf{W}^{\dagger}\right]+\mathbf{Q} .
\end{aligned}
$$

Clearly, the relay's transmit covariance is indeed a complicated function of $\mathbf{W}$. In this letter, we adopt the zeroforcing (ZF) solution constraint to cancel the RSI from the relay output to the relay input via the optimization of $\mathbf{W}$ [4]. In particular, the $\mathrm{ZF}$ constraints may take the following forms [8]

$$
\begin{array}{ll}
\mathbf{W H}_{R R}=\mathbf{0}, & \text { if } M_{R}>M_{T}, \\
\mathbf{H}_{R R} \mathbf{W}=\mathbf{0}, & \text { if } M_{T}>M_{R} .
\end{array}
$$

For convenience, we only consider the case $M_{T}>M_{R}$ as the other case can be handled similarly. Thus, (5) becomes

$$
\begin{array}{r}
\mathbf{x}_{R}[n]=\sqrt{\rho} \mathbf{W}\left[\mathbf{h}_{A R} s_{A}[n-\tau]+\mathbf{h}_{B R} s_{B}[n-\tau]+\mathbf{n}_{R}[n-\tau]\right] \\
+\mathbf{z}[n],
\end{array}
$$

with the relay output power expressed as

$$
\begin{aligned}
P_{R}= & \operatorname{trace}\left(\mathbb{E}\left[\mathbf{x}_{\mathbf{R}} \mathbf{x}_{\mathbf{R}}^{\dagger}\right]\right) \\
=\rho\left[P_{A}\left\|\mathbf{W h}_{A R}\right\|^{2}+P_{B}\left\|\mathbf{W} \mathbf{h}_{B R}\right\|^{2}+\right. & \left.\operatorname{trace}\left(\mathbf{W} \mathbf{W}^{\dagger}\right)\right] \\
& +\operatorname{trace}(\mathbf{Q}) .
\end{aligned}
$$

In the second time slot after cancelling the SI signal $s_{A}[n-\tau]$, the received signal at $S_{A}$ is given as

$$
\begin{array}{r}
y_{S A}[n]=\sqrt{\rho}\left(\mathbf{h}_{R A}^{\dagger} \mathbf{W h}_{B R} s_{B}[n-\tau]+\mathbf{h}_{R A}^{\dagger} \mathbf{W} \mathbf{n}_{R}[n]\right) \\
+\mathbf{h}_{R A}^{\dagger} \mathbf{z}[n]+h_{A A} s_{A}[n]+n_{A}[n],
\end{array}
$$

where $n_{A}[n]$ is the AWGN at node $\mathrm{S}_{\mathrm{A}}$. From this, we can work out the rates at $S_{A}$ and $S_{B}$ as

$$
R_{X}=\log _{2}\left(1+\Gamma_{X}\right), \quad \text { for } X \in\{A, B\},
$$

where

$$
\begin{aligned}
\Gamma_{A} & =\frac{\rho P_{B}\left|\mathbf{h}_{R A}^{\dagger} \mathbf{W} \mathbf{h}_{B R}\right|^{2}}{\rho \sigma_{R}^{2}\left\|\mathbf{h}_{R A}^{\dagger} \mathbf{W}\right\|^{2}+P_{A}\left|h_{A A}\right|^{2}+\mathbf{h}_{R A}^{\dagger} \mathbf{Q} \mathbf{h}_{R A}+1}, \\
\Gamma_{B} & =\frac{\rho P_{A}\left|\mathbf{h}_{R B}^{\dagger} \mathbf{W} \mathbf{h}_{A R}\right|^{2}}{\rho \sigma_{R}^{2}\left\|\mathbf{h}_{R B}^{\dagger} \mathbf{W}\right\|^{2}+P_{B}\left|h_{B B}\right|^{2}+\mathbf{h}_{R B}^{\dagger} \mathbf{Q} \mathbf{h}_{R B}+1} .
\end{aligned}
$$

The signal received at $E$ can be expressed as

$$
\begin{array}{r}
\gamma_{E}[n]=\sqrt{\rho}\left(\mathbf{h}_{R E}^{\dagger} \mathbf{W} \mathbf{h}_{A R} s_{A}[n-\tau]+\mathbf{h}_{R E}^{\dagger} \mathbf{W h}_{B R} s_{B}[n-\tau]\right. \\
\left.+\mathbf{h}_{R E}^{\dagger} \mathbf{W} \mathbf{n}_{R}\right)+\mathbf{h}_{R E}^{\dagger} \mathbf{z}[n]+n_{E},
\end{array}
$$

where $n_{E}$ is the AWGN at E. Also, the achievable sum-rate at $\mathrm{E}$ is upper bounded as $R_{E}=\log _{2}\left(1+\Gamma_{E}\right)$ [8], where

$$
\Gamma_{E}=\frac{\rho P_{A}\left|\mathbf{h}_{R E}^{\dagger} \mathbf{W} \mathbf{h}_{A R}\right|^{2}+\rho P_{B}\left|\mathbf{h}_{R E}^{\dagger} \mathbf{W} \mathbf{h}_{B R}\right|^{2}}{\rho \sigma_{R}^{2}\left\|\mathbf{h}_{R E}^{\dagger} \mathbf{W}\right\|^{2}+\mathbf{h}_{R E}^{\dagger} \mathbf{Q} \mathbf{h}_{R E}+1} .
$$

The achievable secrecy sum-rate is then defined as [8]

$$
R_{s e c}=\left[R_{A}+R_{B}-R_{E}\right]^{+},
$$

where $[x]^{+}$represents $\max (x, 0)$. The eavesdropper is a legitimate user for another service, hence perfect CSI is available. Meanwhile, the signal split to the ER at $R$ is given by

$\mathbf{y}_{R}^{E R}[n]=\sqrt{1-\rho}\left(\mathbf{h}_{A R} s_{A}[n]+\mathbf{h}_{B R} s_{B}[n]+\mathbf{H}_{R R} \mathbf{x}_{R}[n]+\mathbf{n}_{R}[n]\right)$.

The harvested energy at the relay is thus given as [4]

$$
\mathrm{U}=\beta(1-\rho)\left(\left|\mathbf{h}_{A R}\right|^{2} P_{A}+\left|\mathbf{h}_{B R}\right|^{2} P_{B}+\overline{\mathrm{E}}+\sigma_{R}^{2} M_{R}\right),
$$

in which $\overline{\mathrm{E}}=\mathbb{E}\left[\mathbf{x}_{R} \mathbf{x}_{R}^{\dagger}\right]$ and $\beta$ denotes the energy conversion efficiency of the ER at the relay which is assumed unity.

\section{B. Problem Statement}

Due to the inherent SI at each FD node, the nodes may not use the maximum available transmit power in order not to increase the level of SI. Thus, there is a need to transmit at optimum values. Furthermore, it is known that optimal values of system parameters guarantees that the secrecy rate is as large as possible [8]. Thus, in this letter, our aim is to maximize the secrecy sum-rate for SWIPT by ensuring that system parameters are optimal. We achieve this by jointly optimizing the transmit power $\left(P_{A}, P_{B}\right)$ at nodes $\mathrm{S}_{\mathrm{A}}$ and $\mathrm{S}_{\mathrm{B}}$, the 
relaying matrix $(\mathbf{W})$ and the $\mathrm{AN}$ covariance matrix $(\mathbf{Q})$ at the relay. Thus, we have

$$
\begin{gathered}
\min _{\substack{\rho \in(0,1), \mathbf{W}, \mathbf{Q} \geq \mathbf{0} \\
0<P_{A} \leq P_{\max }, 0<P_{B} \leq P_{\max }}} P_{A}+P_{B}+P_{R} \quad \text { s.t. } \\
\left\{\begin{array}{l}
\Gamma_{A} \geq \gamma_{A}, \quad \Gamma_{B} \geq \gamma_{B}, \quad \Gamma_{E} \leq \gamma_{E}, \\
(1-\rho)\left(\left|\mathbf{h}_{A R}\right|^{2} P_{A}+\left|\mathbf{h}_{B R}\right|^{2} P_{B}+\overline{\mathrm{E}}+\sigma_{R}^{2} M_{R}\right) \geq \overline{\mathrm{U}} \\
\mathbf{H}_{R R} \mathbf{W}=\mathbf{0},
\end{array}\right.
\end{gathered}
$$

where $\gamma_{A}, \gamma_{B}, \gamma_{E}$, and $\overline{\mathrm{U}}$ are respective QoS threshold. Note that the second constraint guarantees that the energy receiver circuit can harvest sufficient energy such that the relay can successfully forward the received signals.

\section{PROPOSED SCHEME}

\section{A. Optimization of $\mathbf{W}$ and $\mathbf{Q}$ at the Relay}

Here, we study the optimal beamforming matrix and the AN covariance matrix assuming the transmit power $\left(P_{A}, P_{B}\right)$ and the PS ratio $(\rho)$ all being fixed. For convenience, we define $\mathbf{W}=\mathbf{N}_{t} \mathbf{V}$, where $\mathbf{N}_{t} \in \mathbb{C}^{M_{T} \times M_{T}}$ represents the null space of $\mathbf{H}_{R R}$, and $\mathbf{V} \in \mathbb{C}^{M_{T} \times M_{T}}$ is the new optimization variable. As a consequence, the optimization of $\mathbf{W}$ reduces to optimizing V. Hence, we remove the ZF constraint in (18) and obtain the equivalent optimization problem:

$$
\begin{aligned}
& \min _{\mathbf{V}, \mathbf{Q} \geq \mathbf{0}} P_{R} \quad \text { s.t. } \\
& \left\{\begin{array}{l}
\Gamma_{A} \geq \gamma_{A}, \quad \Gamma_{B} \geq \gamma_{B}, \quad \Gamma_{E} \leq \gamma_{E}, \\
(1-\rho)\left(\left|\mathbf{h}_{A R}\right|^{2} P_{A}+\left|\mathbf{h}_{B R}\right|^{2} P_{B}+\overline{\mathrm{E}}+\sigma_{R}^{2} M_{R}\right) \geq \overline{\mathrm{U}} .
\end{array}\right.
\end{aligned}
$$

Problem (19) is a nonconvex problem due to the coupled optimization variables in the constraints. However, by rearranging the terms in the constraints, (19) can be re-expressed as

$$
\begin{aligned}
& \min _{\Sigma, \mathbf{Q} \geq \mathbf{0}} P_{R} \quad \text { s.t. } \\
& \frac{1}{\gamma_{A}} P_{B} C_{r A} \mathbf{h}_{B R}^{\dagger} \Sigma \mathbf{h}_{B R}-\sigma_{R}^{2} C_{N t} \mathbf{h}_{R A}^{\dagger} \Sigma \mathbf{h}_{R A} \\
& \geq \frac{1}{\rho}\left(P_{A}\left|h_{A A}\right|^{2}+\mathbf{h}_{R A}^{\dagger} \mathbf{Q} \mathbf{h}_{R A}+1\right), \\
& \frac{1}{\gamma_{B}} P_{A} C_{r B} \mathbf{h}_{A R}^{\dagger} \Sigma \mathbf{h}_{A R}-\sigma_{R}^{2} C_{N t} \mathbf{h}_{R B}^{\dagger} \Sigma \mathbf{h}_{R B} \\
& \geq \frac{1}{\rho}\left(P_{B}\left|h_{B B}\right|^{2}+\mathbf{h}_{R B}^{\dagger} \mathbf{Q} \mathbf{h}_{R B}+1\right), \\
& \frac{1}{\gamma_{E}}\left[P_{A} C_{r E} \mathbf{h}_{A R}^{\dagger} \Sigma \mathbf{h}_{A R}+P_{B} C_{r E} \mathbf{h}_{B R}^{\dagger} \Sigma \mathbf{h}_{B R}\right] \\
& -\sigma_{R}^{2} C_{N t} \mathbf{h}_{R E}^{\dagger} \Sigma \mathbf{h}_{R E} \leq \frac{1}{\rho}\left(\mathbf{h}_{R E}^{\dagger} \mathbf{Q} \mathbf{h}_{R E}+1\right), \\
& \left|\mathbf{h}_{A R}\right|^{2} P_{A}+\left|\mathbf{h}_{B R}\right|^{2} P_{B}+\overline{\mathrm{E}} \geq \frac{\mathrm{U}}{(1-\rho)}-\sigma_{R}^{2} M_{R},
\end{aligned}
$$

where $\Sigma=\mathbf{V} \mathbf{V}^{\dagger}, C_{r A}=\left\|\mathbf{N}_{t} \mathbf{h}_{R A}\right\|^{2}, C_{N t}=\operatorname{trace}\left(\mathbf{N}_{t} \mathbf{N}_{t}^{\dagger}\right), C_{r B}=$ $\left\|\mathbf{N}_{t} \mathbf{h}_{R B}\right\|^{2}$ and $C_{r E}=\left\|\mathbf{N}_{t} \mathbf{h}_{R E}\right\|^{2}$. Problem (20) can be efficiently solved by existing solvers such as CVX. Once the optimal $\boldsymbol{\Sigma}$ is obtained, optimal $\mathbf{V}$ can be constructed through matrix decomposition.

\section{B. Optimization of the PS Coefficient $(\rho)$}

For fixed values of the relay beamforming matrix $(\mathbf{W}), \mathrm{AN}$ covariance $(\mathbf{Q})$ and for given values of the transmit power
$\left(P_{A}, P_{B}\right),(18)$ can be reformulated as

$$
\begin{aligned}
& \min _{\rho \in(0,1)} P_{A}+P_{B}+P_{R} \quad \text { s.t. } \\
& \frac{\rho P_{B}\left|\mathbf{h}_{R A}^{\dagger} \mathbf{W} \mathbf{h}_{B R}\right|^{2}}{\rho \sigma_{R}^{2}\left\|\mathbf{h}_{R A}^{\dagger} \mathbf{W}\right\|^{2}+P_{A}\left|h_{A A}\right|^{2}+\mathbf{h}_{R A}^{\dagger} \mathbf{Q} \mathbf{h}_{R A}+1} \geq \gamma_{A}, \\
& \frac{\rho P_{A}\left|\mathbf{h}_{R B}^{\dagger} \mathbf{W h}_{A R}\right|^{2}}{\rho \sigma_{R}^{2}\left\|\mathbf{h}_{R B}^{\dagger} \mathbf{W}\right\|^{2}+P_{B}\left|h_{B B}\right|^{2}+\mathbf{h}_{R B}^{\dagger} \mathbf{Q h} \mathbf{h}_{R B}+1} \geq \gamma_{B}, \\
& \frac{\rho P_{A}\left|\mathbf{h}_{R E}^{\dagger} \mathbf{W} \mathbf{h}_{A R}\right|^{2}+\rho P_{B}\left|\mathbf{h}_{R E}^{\dagger} \mathbf{W h}_{B R}\right|^{2}}{\rho \sigma_{R}^{2}\left\|\mathbf{h}_{R E}^{\dagger} \mathbf{W}\right\|^{2}+\mathbf{h}_{R E}^{\dagger} \mathbf{Q} \mathbf{h}_{R E}+1}, \\
& (1-\rho)\left(\left|\mathbf{h}_{A R}\right|^{2} P_{A}+\left|\mathbf{h}_{B R}\right|^{2} P_{B}+\overline{\mathrm{E}}+\sigma_{R}^{2} M_{R}\right) \geq \overline{\mathrm{U}},
\end{aligned}
$$

which can be expressed in a form solvable by existing solvers by rearranging the terms in the constraints as

$$
\begin{aligned}
& \min _{\rho \in\{0,1\}} P_{A}+P_{B}+P_{R} \quad \text { s.t. } \\
& \frac{1}{\gamma_{A}} \rho P_{B} C_{r A} \mathbf{h}_{B R}^{\dagger} \Sigma \mathbf{h}_{B R}-\rho \sigma_{R}^{2} C_{N t} \mathbf{h}_{R A}^{\dagger} \Sigma \mathbf{h}_{R A} \\
& \geq P_{A}\left|h_{A A}\right|^{2}+\mathbf{h}_{R A}^{\dagger} \mathbf{Q h} \mathbf{h}_{R A}+1, \\
& \frac{1}{\gamma_{B}} \rho P_{A} C_{r B} \mathbf{h}_{A R}^{\dagger} \Sigma \mathbf{h}_{A R}-\rho \sigma_{R}^{2} C_{N t} \mathbf{h}_{R B}^{\dagger} \Sigma \mathbf{h}_{R B} \\
& \geq P_{B}\left|h_{B B}\right|^{2}+\mathbf{h}_{R B}^{\dagger} \mathbf{Q h} \mathbf{h}_{R B}+1, \\
& \frac{1}{\gamma_{E}}\left[P_{A} C_{r E} \mathbf{h}_{A R}^{\dagger} \Sigma \mathbf{h}_{A R}+P_{B} C_{r E} \mathbf{h}_{B R}^{\dagger} \Sigma \mathbf{h}_{B R}\right] \\
& -\sigma_{R}^{2} C_{N t} \mathbf{h}_{R E}^{\dagger} \Sigma \mathbf{h}_{R E} \leq \frac{1}{\rho}\left(\mathbf{h}_{R E}^{\dagger} \mathbf{Q} \mathbf{h}_{R E}+1\right), \\
& (1-\rho)\left(\left|\mathbf{h}_{A R}\right|^{2} P_{A}+\left|\mathbf{h}_{B R}\right|^{2} P_{B}+\overline{\mathrm{E}}+\sigma_{R}^{2} M_{R}\right) \geq \overline{\mathrm{U}} \text {. }
\end{aligned}
$$

C. Optimization of the Source Power $\left(P_{A}, P_{B}\right)$

For given values of the relay beamforming matrix $(\mathbf{W}), \mathrm{AN}$ covariance matrix $(\mathbf{Q})$ and the relay PS ratio, problem (18) can be written as

$$
\begin{aligned}
& \min _{P_{A}, P_{B}} P_{A}+P_{B}+P_{R} \quad \text { s.t. } \\
& \frac{\rho P_{B}\left|\mathbf{h}_{R A}^{\dagger} \mathbf{W h}_{B R}\right|^{2}}{\rho \sigma_{R}^{2}\left\|\mathbf{h}_{R A}^{\dagger} \mathbf{W}\right\|^{2}+P_{A}\left|h_{A A}\right|^{2}+\mathbf{h}_{R A}^{\dagger} \mathbf{Q} \mathbf{h}_{R A}+1} \geq \gamma_{A}, \\
& \frac{\rho P_{A}\left|\mathbf{h}_{R B}^{\dagger} \mathbf{W h}_{A R}\right|^{2}}{\rho \sigma_{R}^{2}\left\|\mathbf{h}_{R B}^{\dagger} \mathbf{W}\right\|^{2}+P_{B}\left|h_{B B}\right|^{2}+\mathbf{h}_{R B}^{\dagger} \mathbf{Q} \mathbf{h}_{R B}+1} \geq \gamma_{B}, \\
& \frac{\rho P_{A}\left|\mathbf{h}_{R E}^{\dagger} \mathbf{W} \mathbf{h}_{A R}\right|^{2}+\rho P_{B}\left|\mathbf{h}_{R E}^{\dagger} \mathbf{W h}_{B R}\right|^{2}}{\rho \sigma_{R}^{2}\left\|\mathbf{h}_{R E}^{\dagger} \mathbf{W}\right\|^{2}+\mathbf{h}_{R E}^{\dagger} \mathbf{Q} \mathbf{h}_{R E}+1}, \\
& (1-\rho)\left(\left|\mathbf{h}_{A R}\right|^{2} P_{A}+\left|\mathbf{h}_{B R}\right|^{2} P_{B}+\overline{\mathrm{E}}+\sigma_{R}^{2} M_{R}\right) \geq \overline{\mathrm{U}}, \\
& 0<P_{A} \leq P_{\max }, \quad 0<P_{B} \leq P_{\max } .
\end{aligned}
$$

It is worth noting that full-duplexity in communication systems is preceded by successful SI cancellation. In our model, both $\mathrm{S}_{\mathrm{A}}$ and $\mathrm{S}_{\mathrm{B}}$ are equipped with a single transmitter-receiver pair for signal transmission and reception, respectively. As a result, it is impossible to cancel the SI in the spatial domain [4]. The relay, in contrast, equipped with at least two transmitterreceiver pairs, can cancel the generated SI in the spatial domain. We proceed to investigate the optimal power solution $\left(P_{A}, P_{B}\right)$ assuming $\mathbf{W}, \mathbf{Q}$ and $\rho$ all being fixed. Evidently, it is easy to check that at the optimum, either $\mathrm{S}_{\mathrm{A}}$ or $\mathrm{S}_{\mathrm{B}}$ will be transmitting with maximum power [4], i.e., $P_{A}=P_{\max }$ or $P_{B}=P_{\max }$. As a consequence, we can relax (23) into two subproblems with: (i) $P_{A}=P_{\max }$, (ii) $P_{B}=P_{\max }$. Considering the symmetric nature of case (i) and case (ii), we study case (i) as 


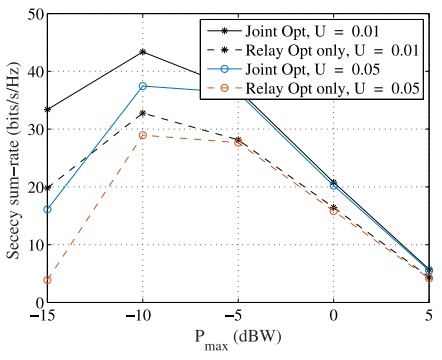

Fig. 1. Secrecy sum-rate versus $P_{\max }$.

an example and solve problem (23) analytically. Problem (23) is thus reformulated as

$$
\begin{aligned}
& \min _{P_{B}} P_{B}+\bar{P}_{R} \quad \text { s.t. } \\
& \frac{\rho P_{B}\left|\mathbf{h}_{R A}^{\dagger} \mathbf{W} \mathbf{h}_{B R}\right|^{2}}{\rho \sigma_{R}^{2}\left\|\mathbf{h}_{R A}^{\dagger} \mathbf{W}\right\|^{2}+P_{\max }\left|h_{A A}\right|^{2}+\mathbf{h}_{R A}^{\dagger} \mathbf{Q} \mathbf{h}_{R A}+1} \geq \gamma_{A}, \\
& \frac{\rho P_{\max }\left|\mathbf{h}_{R B}^{\dagger} \mathbf{W} \mathbf{h}_{A R}\right|^{2}}{\rho \sigma_{R}^{2}\left\|\mathbf{h}_{R B}^{\dagger} \mathbf{W}\right\|^{2}+P_{B}\left|h_{B B}\right|^{2}+\mathbf{h}_{R B}^{\dagger} \mathbf{Q} \mathbf{h}_{R B}+1} \geq \gamma_{B}, \\
& \frac{\rho P_{\max }\left|\mathbf{h}_{R E}^{\dagger} \mathbf{W} \mathbf{h}_{A R}\right|^{2}+\rho P_{B}\left|\mathbf{h}_{R E}^{\dagger} \mathbf{W} \mathbf{h}_{B R}\right|^{2}}{\rho \sigma_{R}^{2}\left\|\mathbf{h}_{R E}^{\dagger} \mathbf{W}\right\|^{2}+\mathbf{h}_{R E}^{\dagger} \mathbf{Q h}} \leq \gamma_{R E}+1 \\
& (1-\rho)\left(\left|\mathbf{h}_{A R}\right|^{2} P_{\max }+\left|\mathbf{h}_{B R}\right|^{2} P_{B}+\overline{\mathrm{E}}+\sigma_{R}^{2} M_{R}\right) \geq \overline{\mathbf{U}}, \\
& 0<P_{B} \leq P_{\max },
\end{aligned}
$$

where $\bar{P}_{R}=\rho\left[P_{\max }\left\|\mathbf{W} \mathbf{h}_{A R}\right\|^{2}+P_{B}\left\|\mathbf{W} \mathbf{h}_{B R}\right\|^{2}+\operatorname{trace}\left(\mathbf{W} \mathbf{W}^{\dagger}\right)\right]+$ $\operatorname{trace}(\mathbf{Q})$. Since $0<P_{B} \leq P_{\max }$, we can obtain the feasible range $\left[P_{B}^{\min }, P_{B}^{\max }\right]$ for $P_{B}$. Also, the constraints in (24) can be analysed with respect to $P_{B}$ :

1) A continuous increase in $P_{B}$ should guarantee that (24b) remains satisfied. As a consequence, we can set the minimum of $P_{B}$ as $P_{B}^{\min }=\frac{\gamma_{A}\left(\rho \sigma_{R}^{2}\left\|\mathbf{h}_{R A}^{\dagger} \mathbf{W}\right\|^{2}+P_{A}\left|h_{A A}\right|^{2}+\mathbf{h}_{R A}^{\dagger} \mathbf{Q h} \mathbf{h}_{R A}+1\right)}{\rho\left|\mathbf{h}_{R A}^{\dagger} \mathbf{W h}_{B R}\right|^{2}}$.

2) Constraint $(24 \mathrm{c})$ is a decreasing function of $P_{B}$. Therefore, the maximum $P_{B}$ satisfying (24c) to equality is defined as $P_{B}^{\max }=\frac{\rho P_{A}\left|\mathbf{h}_{R B}^{\dagger} \mathbf{W} \mathbf{h}_{A R}\right|^{2}-\gamma_{B}\left(\rho \sigma_{R}^{2}\left\|\mathbf{h}_{R B}^{\dagger} \mathbf{W}\right\|^{2}+\mathbf{h}_{R B}^{\dagger} \mathbf{Q h} h_{R B}+1\right)}{\gamma_{B}\left|h_{B B}\right|^{2}}$.

3) An upper bound of the eavesdropping constraint in (24d) is satisfied when $P_{B} \leq P_{B}^{\max }$.

4) A lower bound of the energy harvesting constraint in (24e) is guaranteed to be satisfied when $P_{B} \geq P_{B}^{\min }$.

The optimal $P_{B}^{*}$ is chosen between $P_{B}^{\min }$ and $P_{B}^{\max }$ which satisfies (24b)-(24e). Accordingly, to obtain the optimal $P_{B}$, we perform a 1-D search over $P_{B}$ starting from $P_{B}^{\min }$ until $P_{B}^{\max }$ is reached to find a feasible solution to problem (24). Clearly, if $P_{B}^{\min }>P_{B}^{\max }$ then (24) becomes infeasible.

Thus problem (18) is solved in an alternating manner.

\section{Simulation Results}

In this section, we consider Rayleigh flat fading channels for computer simulations. The results are averaged over 1000 channel realizations with $\gamma_{A}=-5(\mathrm{~dB}), \gamma_{B}=-5(\mathrm{~dB}), \gamma_{E}=$ $-15(\mathrm{~dB})$. We also assume that $60 \%$ of the SI at node A and node $B$ has been eliminated through digital cancellation [4].

In Fig. 1, we investigate the secrecy sum-rate for SWIPT in FD systems versus the transmit power budget $P_{\max }(\mathrm{dB})$ for different values of the harvested power constraint. We study the performance of the proposed scheme (denoted 'Joint Opt.' in the figure) in comparison with the relay-only optimization scheme (denoted 'Relay Only Opt.'). We see that the proposed scheme yields a higher secrecy sum-rate than the relay-only optimization scheme. Also, it can be observed that the secrecy

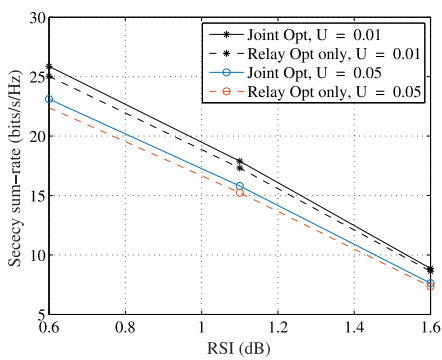

Fig. 2. Secrecy sum-rate versus RSI.

sum-rate increases with $P_{\max }$ to a certain level after which it begins to experience a decrease with a continuous increase in $P_{\max }$, due to the increase of RSI [10].

In Fig. 2, we investigate further the secrecy sum-rate performance against the RSI for different values of the harvested power constraints. Evidently, as RSI increases, a corresponding decrease in the secrecy sum-rate is observed. However, the proposed scheme yields higher secrecy sumrate compared to the secrecy sum-rate of the relay-only optimization scheme for different values of the harvested energy constraint. Hence, the need for joint optimization is justified.

Note from Fig. 1 and 2, as the harvested energy threshold increases, more power is directed to the ER of the relay. The result is a corresponding decline in the achievable secrecy rate.

\section{CONClusion}

This letter investigated the joint optimization of the transmit power, AN covariance matrix, and the relay beamforming matrix for SWIPT in FD AF relaying system in the presence of an eavesdropper. Specifically, using SDP and 1-D searching, we proposed an algorithm that minimizes the total transmit power for secure SWIPT in a FD MIMO AF relay system.

\section{REFERENCES}

[1] R. Zhang and C. K. Ho, "MIMO broadcasting for simultaneous wireless information and power transfer," IEEE Trans. Wireless Commun., vol. 12, no. 5, pp. 1989-2001, May 2013.

[2] M. Duarte and A. Sabharwal, "Full-duplex wireless communications using off-the-shelf radios: Feasibility and first results," in Proc. IEEE Asilomar, Pacific Grove, CA, USA, 2010, pp. 1558-1562.

[3] L. Lai and H. E. Gamal, "The relay-Eavesdropper channel: Cooperation for secrecy," IEEE Trans. Inf. Theory, vol. 54, no. 9, pp. 4005-4019, Sep. 2008.

[4] A. A. Okandeji, M. R. A. Khandaker, K.-K. Wong, and Z. Zheng, "Joint transmit power and relay two-way beamforming optimization for energyharvesting full-duplex communications," in Proc. IEEE Globecom Int. Workshop Full Duplex Wireless Commun., Washington, DC, USA, Dec. 2016, pp. 1-6.

[5] Y. Wang, R. Sun, and X. Wang, "Transceiver design to maximize the weighted sum secrecy rate in full-duplex SWIPT systems," IEEE Signal Process. Lett., vol. 23, no. 6, pp. 883-887, Jun. 2016.

[6] Y. Wan, Q. Li, Q. Zhang, and J. Qin, "Optimal and suboptimal full-duplex secure beamforming designs for MISO two-way communications," IEEE Wireless Commun. Lett., vol. 4, no. 5, pp. 493-496, Oct. 2015.

[7] M. R. A. Khandaker, K.-K. Wong, and G. Zheng, "Truth-telling mechanism for two-way relay selection for secrecy communications with energy-harvesting revenue," IEEE Trans. Wireless Commun., vol. 16, no. 5, pp. 3111-3123, May 2017.

[8] Q. Li and D. Han, "Sum secrecy rate maximization for full-duplex twoway relay networks," in Proc. IEEE Int. Conf. Acoust. Speech Signal Process., Shanghai, China, Mar. 2016, pp. 3641-3645.

[9] A. E. Gamal, N. Hassanpour, and J. Mammen, "Relay networks with delays," IEEE Trans. Inf. Theory, vol. 53, no. 10, pp. 3413-3431, Oct. 2007.

[10] Q. Li, W.-K. Ma, and D. Han, "Sum secrecy rate maximization for full-duplex two-way relay networks using Alamouti-based rank-two beamforming," IEEE J. Sel. Topics Signal Process., vol. 10, no. 8, pp. 1359-1374, Dec. 2016. 